\title{
Permanencia estudiantil en el curso Matemática General de la Universidad Nacional, Costa Rica
}

Student permanence in the Matematica General course of the Universidad Nacional, Costa Rica

\author{
Volumen 20, Número 1 \\ Enero - Abril \\ pp. 1-21
}

José Andrey Zamora Araya

Ronny Gamboa Araya

Randall Hidalgo Mora

Mario Castillo Sánchez

Citar este documento según modelo APA

Zamora Araya, José Andrey; Gamboa Araya, Ronny; Hidalgo Mora, Randall y Castillo Sánchez Mario. (2020). Permanencia estudiantil en el curso Matemática General de la Universidad Nacional, Costa Rica. Revista Actualidades Investigativas en Educación, 20(1), 1-21. Doi. 10.15517/aie.v20i1.39815 


\title{
Permanencia estudiantil en el curso Matemática General de la Universidad Nacional, Costa Rica
}

Student permanence in the Matematica General course of the Universidad Nacional, Costa Rica

\author{
José Andrey Zamora Araya ${ }^{1}$ \\ Ronny Gamboa Araya ${ }^{2}$ \\ Randall Hidalgo Mora ${ }^{3}$ \\ Mario Castillo Sánchez ${ }^{4}$
}

\begin{abstract}
Resumen: El abandono estudiantil es un fenómeno de carácter multifactorial que afecta sobre todo a poblaciones en condición de vulnerabilidad. Dado que el abandono se da en una mayor proporción en los primeros semestres, son los cursos introductorios los más afectados, y esta situación se aprecia, en particular, en el área de Matemática. Este artículo muestra los principales resultados de un estudio cuantitativo cuyo objetivo fue determinar, mediante un análisis de regresión logística múltiple, cuáles son los factores más relevantes relacionados con el abandono escolar en el curso de Matemática General de la Universidad Nacional, Costa Rica. La información fue obtenida mediante un cuestionario auto-administrado a 906 personas estudiantes matriculadas en el curso durante el primer ciclo de 2018. Los resultados muestran que la tenencia de beca y el rendimiento previo en secundaria actúan como factores protectores. Por su parte, la condición laboral, pertenecer a carreras del área de ingeniería, ser hombre y el estrato de procedencia se consideran factores de riesgo. Es necesario que la Universidad tome acciones que procuren aumentar los niveles de permanencia del estudiantado proveniente de contextos con menores oportunidades educativas, que brinden no solo acceso, sino acompañamiento durante el primer año de carrera. Para ello, se sugiere coordinar con otras instancias universitarias, establecer un programa de tutorías y evaluar un rediseño de contenidos del curso de Matemática General.
\end{abstract}

Palabras clave: permanencia escolar, educación superior, enseñanza de las matemáticas

\begin{abstract}
Scholar dropout affects vulnerable condition populations and has a multifactorial character. Dropout occurs in a greater proportion in the first semesters, the introductory courses are the most affected, and this situation is particularly observed in the area of Mathematics. This article shows the main results about a quantitate study whose aims was determine, by means of a multiple logistic regression analysis, which are the most relevant factors related to school dropout in the Matematica General course of the Universidad Nacional, Costa Rica. The information was obtained through a self-administered questionnaire to 906 students enrolled in the course during the first semester of 2018. The results show that the scholarship holding and the previous academic performance in high school act as protective factors; On the other hand, the laboral condition, belonging to careers in the engineering area, being a man and the group of origin are considered risk factors. It is necessary that university take actions that seek to increase the permanence of students from contexts with educational disadvantages, providing not only access but accompaniment during the first year of the career; for which it is suggested to coordinate with other university instances such as the mentoring program as well as to evaluate a redesign of contents of the Matematica General course.
\end{abstract}

Keys Words: school stay, higher education, mathematics education.

\footnotetext{
${ }_{1}^{1}$ Investigador y académico de la Universidad Nacional, Costa Rica, en la Escuela de Matemática. Dirección electrónica: izamo@una.ac.cr Orcid http://orcid.org/0000-0001-6050-5850

2 Investigador y académico de la Universidad Nacional, Costa Rica, en la Escuela de Matemática. Dirección electrónica: ronny.gamboa.araya@una.cr Orcid http://orcid.org/0000-0002-9531-0372

${ }^{3}$ Investigador y académico de la Universidad Nacional, Costa Rica, en la Escuela de Matemática. Dirección electrónica: randall.hidalgo.mora@una.cr Orcid http://orcid.org/0000-0002-8405-1600

4 Investigador y académico de la Universidad Nacional, Costa Rica, en la Escuela de Matemática. Dirección electrónica: mario.castillo.sanchez@una.cr Orcid http://orcid.org/0000-0002-2078-0628
}

Artículo recibido: 19 de junio, 2019

Enviado a corrección: 24 de setiembre, 2019

Aprobado: 14 de octubre, 2019 


\section{Introducción}

El abandono en los primeros cursos de matemática, a nivel universitario, es una problemática que posee diversas implicaciones: económicas, tanto para el individuo como para las instituciones de educación superior; académicas, como el atraso o rezago en el plan de estudios y periodo de graduación; y personales, como la motivación, estrés, entre otros (Crawford, 2014; Esteban, Bernardo y Rodríguez-Muñiz, 2016).

A pesar de la extensa literatura enfocada en el análisis del abandono universitario en la disciplina, no es posible determinar un único factor asociado, sino que es un fenómeno multifactorial (Canales y De los Ríos, 2007). Factores como la formación académica previa y el aprendizaje adquirido, el ambiente educativo, cursos teóricos y desvinculados del mercado laboral, la edad, el proceso de adaptación a la vida universitaria, el cambio del modelo pedagógico, tipos de evaluación, cursos de poco interés para su ejercicio profesional, entre otros han sido mencionados por algunas personas investigadoras como aspectos relacionados con esta problemática (González, Uribe y González, 2005; Páramo y Correa, 1999).

Particularmente en la Universidad Nacional (UNA), el curso de Matemática General, ofertado por la Escuela de Matemática, es el primero del área a nivel universitario para la mayoría del estudiantado, no obstante, el rendimiento en él es bajo. Por ejemplo, con base en la información recolectada en las actas de calificaciones de la Escuela de Matemática, se determinó que durante el I semestre del año 2018 tan solo el 31,1\% lo aprobó y el 36,3\% del estudiantado ni siquiera concluyó el curso. A su vez, en el Il semestre los porcentajes fueron de $35,8 \%$ y $38,5 \%$, respectivamente.

Los datos anteriores son reflejo del abandono que se presenta en los cursos iniciales del área a nivel universitario. Cabe destacar que el abandono prematuro de los estudios es uno de los principales problemas que afronta la educación superior y que impacta en diferentes ámbitos, tanto social como institucional y personal. En el plano social, disminuye la posibilidad de lograr un mejor status e incrementa el costo de la educación al no poder optimizar los recursos disponibles; en el ámbito institucional, afecta el cumplimiento de los objetivos de la universidad, baja los índices de eficiencia, calidad e ingreso; y en lo personal, aumenta la sensación de fracaso escolar, reduce las posibilidades de obtener un trabajo bien remunerado y aumenta el tiempo de graduación y permanencia en la institución (International Institute for Higher Education in Latin America, 2006; Rodríguez-Pineda, 2018). 
En el caso del curso Matemática General de la UNA, existen factores de tipo académico, social y económico que podrían estar relacionados con el abandono escolar. Además, aspectos como sexo, edad, condición de beca, condición de trabajo, estrato, tipo de colegio, nota de colegio, nota de ingreso a la universidad, carrera que cursa la persona estudiante, entre otros se identificaron como posibles factores asociados al abandono del curso (Rodríguez-Pineda, 2018). A partir de lo anterior, surgen ocho hipótesis de trabajo de cómo estos factores pueden estar relacionados con la magnitud del fenómeno. Los planteamientos de estas hipótesis surgen a partir de los resultados señalados por otras personas investigadoras. Por ejemplo, se piensa que el estudiantado que trabaja, de mayor edad, proveniente de estratos bajos, que son hombres y estudian carreras del área de las ingenierías tiene mayor tasa de abandono (Avdic y Gartell, 2015; Crawford, 2014; Gessaghi y Llinás, 2005, Himmel, 2002; Tinto, 1982).

Asimismo, tener beca y buenos puntajes en las notas de la educación secundaria y en el examen de admisión se consideran como factores que posibilitan la permanencia en las aulas universitarias, lo cual se apoya tanto en estudios realizados en el contexto de la UNA como a nivel internacional (Canales y De los Ríos, 2007; Esteban et al., 2016; Ramírez, 2013; Rodríguez-Pineda y Zamora-Araya, 2014a).

El presente artículo pretende, por medio de un análisis de regresión logística, determinar si variables como el sexo, la beca, la condición laboral, el estrato, la nota de admisión, la nota de colegio, la edad y la carrera pueden ser consideradas como factores de riesgo o de protección para el abandono en el curso Matemática General.

\section{Referente teórico}

\subsection{Abandono estudiantil}

En América Latina, a pesar de los procesos de democratización experimentados en el acceso a la educación superior, en particular desde la segunda mitad del siglo XX, no ha sido posible cumplir a cabalidad con dos de sus principales objetivos: promover una mayor justicia social y disminuir la desigualdad determinada por las condiciones sociales relacionadas con el origen del estudiantado (Gessaghi y Llinás, 2005). No obstante, el hecho de que las instituciones de educación superior cuenten con una población cada vez más heterogénea respecto al estrato socioeconómico, área de procedencia y conocimientos previos, ha traído consigo una serie de retos, uno de los principales es la reducción de las tasas de abandono. 
La investigación sobre temas de abandono es extensa, según Braxton, Milem y Sullivan (2000) hay cinco enfoques desde los cuales se puede explicar el fenómeno: el psicológico, sociológico, economicista, organizacional e interaccionista. Adicionalmente, existe un sexto enfoque llamado holístico o integrador que toma en cuenta un gran número de variables explicativas sobre el abandono, cuyas interrelaciones deben ser analizadas considerando tanto los rasgos individuales como el contexto donde se presentan (Torres, 2010).

Numerosos estudios han identificado, basados en uno o más de los enfoques anteriores, factores asociados al abandono estudiantil a nivel universitario, entre los que se pueden citar: el ingreso económico, sexo de la persona estudiante, condición laboral, la motivación, rendimiento académico previo, rendimiento académico en la universidad, asistencia a clase, relación con el profesorado, entre otros (Burbano, Lozano, Medina y Guerrero, 2015; Díaz-Mujica, García, López, Maluenda, Hernández y Pérez-Villalobos, 2018; Rodríguez-Pineda y Zamora-Araya, 2014a; Sandoval, Sánchez, Velasteguí y Naranjo, 2018; Santos, 2009).

Otro factor relacionado con el abandono es el tipo de carrera o titulación, pues existen diferencias en las tasas de abandono según el área de conocimiento del programa académico en que se ubique la persona estudiante (Campillo, García, Hernández, Martínez y Sánchez, 2018; Rodríguez-Pineda y Zamora-Araya, 2014b; Zamora-Araya y VillalobosMadrigal, 2018a). Asimismo, diferentes estudios han modelado el abandono escolar utilizando modelos matemáticos multivariables, tales como la regresión logística, análisis de supervivencia, análisis discriminante, entre otros (Cabrera, Nora, Castaneda, 1993; Castaño, Gallón, Gómez, y Vásquez, 2006; Henríquez y Escobar, 2016), cuyos resultados son acordes con las investigaciones anteriormente mencionadas.

Por otra parte, cuando se habla de abandono escolar, el fenómeno se acentúa en aquellas personas provenientes de los sectores más vulnerables de la sociedad, ya que presupone un costo económico, una decepción anímica y, para las familias más necesitadas, significa la imposibilidad de reincorporarse a los estudios aumentando con ello la brecha de desigualdad en la sociedad (Avdic y Gartell, 2015; Canales y De los Ríos, 2018; Crawford, 2014). Por consiguiente, resulta relevante investigar los posibles factores relacionados con el abandono escolar, a nivel universitario, como medio para gestionar acciones tendientes a su reducción y, a la vez, aumentar las posibilidades de graduación, especialmente para que el 
estudiantado de los sectores más desfavorecidos tenga una oportunidad real de ascenso social.

En resumen, el abandono es un fenómeno complejo y multifactorial, esto se evidencia a la hora de tratar de definirlo. Existen varios conceptos del término, pero como lo señala Tinto (1982) es difícil llegar a un consenso, por lo que este autor considera la imposibilidad de brindar una definición que englobe la totalidad del concepto. De ahí que las personas investigadoras deban seleccionar aquella que mejor se adapte a sus objetivos e intereses.

Con base en lo anterior, para los fines de este estudio, se considera que una persona estudiante matriculada en el curso Matemática General ha abandonado la asignatura si no asiste a la aplicación de alguno de los tres exámenes parciales y los subsiguientes. En este sentido, se considera que una persona estudiante ha abandonado el curso si:

a) no realizó ninguna prueba parcial;

b) realizó la primera prueba parcial pero no se presentó ni a la segunda ni a la tercera;

c) no se presentó a realizar la tercera prueba parcial a pesar de haber realizado las dos anteriores.

\subsection{Abandono en la UNA}

Existen algunos estudios que se han realizado sobre el abandono en la UNA. A nivel institucional, los trabajos de Rodríguez-Pineda y Zamora-Araya (2014a, 2014b) logran establecer una tipología que permite registrar la permanencia del alumnado en seis categorías, que son: población graduada en el tiempo establecido, población graduada con rezago, población egresada, población rezagada activa, desertor temprano y desertor itinerante. En lo que respecta al abandono, las personas investigadoras muestran que, a lo largo de la trayectoria del estudiantado, en términos generales, cerca del $50 \%$ de ellos abandonan los estudios, la mayor proporción del fenómeno ocurre durante el primer año. Además, encuentran que los principales factores asociados con la permanencia son la tenencia de beca, el porcentaje de créditos aprobados, la nota del examen de admisión, la nota de colegio (como factores protectores) y la edad (como factor de riesgo).

Por su parte, otros estudios se concentran en identificar factores relacionados con el fenómeno en programas de estudio particulares. Por ejemplo, Ramírez (2013) efectuó una descripción de la deserción en la UNA y enfatizó su análisis en la carrera de Psicología, durante los años 2007 al 2011, mediante entrevistas telefónicas que le permitieron abordar variables de tipo institucional e individual. Otros estudios sobre carreras en la UNA, 
particularmente de la carrera de Enseñanza de la Matemática, son el de Pascua-Cantero (2016), quien, por medio de entrevistas semi-estructuradas, concluye que la falta de preparación para llevar cursos de matemática, el ambiente de aula, la poca flexibilidad en los horarios, las dificultades para comprender la materia, aspectos económicos y expectativas no satisfechas acerca de la carrera fueron factores que incidieron en el abandono.

Aunado a las investigaciones anteriores, el estudio de Zamora-Araya y VillalobosMadrigal (2018a) describió las características del estudiantado de primer ingreso de la carrera de Enseñanza de Matemática para el año 2016, tanto desertores como no desertores, el estudio muestra que las mujeres, las personas residentes de zonas urbanas y no becadas son las que abandonan la carrera en mayor proporción; además, concluyeron que las variables más relevantes fueron cantidad de créditos matriculados, nota de admisión y el número de créditos aprobados.

Más recientemente, Gamboa, Castillo e Hidalgo (2019) realizaron un estudio descriptivo sobre el rendimiento académico relacionado con los errores que comete el estudiantado en la prueba de diagnóstico en la UNA y que se reflejan en los cursos iniciales que la Escuela de Matemática ofrece a la comunidad universitaria. Las personas investigadoras concluyeron que existe una brecha importante en cuanto a contenidos entre la educación secundaria y la educación universitaria en el área de Matemática. Esto se refleja en los índices de aprobación y abandono en dichos cursos, en particular Matemática General. Sin embargo, hasta la fecha, no se han realizados estudios a profundidad sobre los factores asociados con el abandono escolar en el curso de Matemática General de la UNA. El presente estudio pretende brindar información sobre el tema del abandono estudiantil en el curso de Matemática General para la toma de decisiones tendientes a disminuir la magnitud del fenómeno.

\subsection{Proceso de admisión y estratificación en la UNA}

Desde sus inicios en 1973, la UNA ha cambiado su sistema de admisión en varias ocasiones y ha utilizado diferentes criterios para seleccionar a las personas estudiantes de nuevo ingreso. El sistema actual, implementado desde el año 2009, se conoce como modelo de estratificación y tipificación, el cual incorpora un "factor técnico" constituido por la prueba de actitud académica (PAA) construida por la Universidad de Costa Rica (UCR) y las notas del ciclo diversificado de educación secundaria, y un "factor social" que toma variables del contexto (modalidad del colegio, tipo de financiamiento, horario lectivo y ubicación 
geográfica), las cuales inciden en el rendimiento académico del estudiantado y están relacionadas con la accesibilidad a la educación secundaria (Rodríguez-Pineda, 2018).

Es así como mediante una ponderación del $60 \%$ de la PAA y un $40 \%$ de las notas de educación diversificada se obtiene la así llamada nota ponderada o nota de admisión. Una vez obtenida esa nota, se aplica un proceso denominado estratificación, que toma en cuenta las variables relacionadas con el factor social y que determina la composición de tres estratos (Universidad Nacional [UNA], 2009), los cuales se especifican a continuación:

a) Estrato l: estudiantes provenientes de colegios científicos, subvencionados, humanísticos, privados y extranjeros.

b) Estrato II: estudiantes de colegios públicos de la modalidad académica o técnica profesional diurnos.

c) Estrato III: estudiantes que proceden de instituciones consideradas con menores oportunidades educativas e incluyen modalidades o instituciones como colegio nocturno, bachillerato por madurez, educación abierta, Centro Integrado de Educación de Adultos (CINDEA), Institutos Profesionales de Educación Comunitaria (IPEC), liceos rurales, colegios ubicados en zonas indígenas, telesecundarias, bachillerato a distancia, plan de estudios para la educación de jóvenes y adultos, nuevas oportunidades educativas para jóvenes, entre otros.

El siguiente paso es obtener el promedio $\mu$ y la desviación estándar $\sigma$ de la nota de admisión de todo el estudiantado que realizó la prueba y los $\mu_{i}$ y $\sigma_{i}$ correspondientes a cada estrato $(i=1,2,3)$. Posteriormente, se aplica una técnica denominada tipificación, basada en el hecho de que la nota de admisión tiene una distribución aproximadamente normal, la cual consiste en restarle a la nota de admisión de la persona estudiante la nota promedio del estrato al que pertenece y dividirlo entre la desviación estándar del estrato en cuestión. A estos valores se les denomina puntajes $Z_{i}$, que son la base para obtener la nota tipificada (Rodríguez-Pineda, 2018).

La nota tipificada de una persona estudiante $i$ se obtiene al multiplicar su puntaje $Z_{i}$ por la desviación estándar de la nota de admisión de toda la población y sumarle el promedio de la nota de admisión poblacional. Finalmente, se compara la nota tipificada con la nota de admisión seleccionando la mayor de ellas, y esa es la nota con la cual las personas estudiantes compiten por los cupos a las diferentes carreras de la UNA. 
La intención de este modelo de estratificación es que las mejores personas estudiantes de los estratos II y III (poblaciones con menores oportunidades educativas y provenientes de contextos sociales más vulnerables) puedan competir de manera más equitativa con el estudiantado del estrato I por los cupos que ofrece la UNA (Rodríguez-Pineda, 2018).

\section{Metodología}

\subsection{Enfoque y diseño de la investigación}

De acuerdo con Villalobos (2017), esta investigación se enmarca dentro de un enfoque cuantitativo de diseño no experimental, correlacional de corte transversal. El propósito de la aplicación del modelo es de tipo explicativo y no predictivo, pues la idea es determinar los principales factores asociados con el abandono estudiantil de la población estudiada, para, poder así, plantear recomendaciones que permitan reducir esta problemática.

Además, el análisis de la información pretende contrastar las siguientes hipótesis basadas en la literatura anteriormente planteada, y relacionadas con el curso de Matemática General en la UNA:

1. El estudiantado que posee trabajo tiene mayor riesgo de abandono.

2. El estudiantado que matricula carreras del área de ingeniería tiene mayor riesgo de abandono.

3. Los hombres presentan un mayor riesgo de abandono que las mujeres.

4. La tenencia de beca reduce el riesgo de abandono.

5. Cuanto mayor sea la nota del examen de admisión a la universidad menor es el riesgo de abandono.

6. Entre mayor sea la nota del colegio menor es el riesgo de abandono.

7. El estudiantado perteneciente al estrato III presenta mayor riesgo de abandono en comparación con los otros estratos.

8. Cuanto mayor sea la edad del estudiantado mayor es el riesgo de abandono.

\subsection{Población de estudio}

La población considerada para este estudio consistió en la totalidad del estudiantado matriculado en el curso Matemática General en el primer semestre del año 2018 pertenecientes a carreras de áreas como: química, ciencias económicas, educación, ingenierías y geografía. Se tomaron como criterios de inclusión que la persona estudiante estuviera dispuesta a brindar la información y que se tuviera información completa de todas 
las variables provistas por el Departamento de Registro de la UNA. Como criterios de exclusión se utilizó que la persona estudiante ingresara a la universidad antes del año 2016 o que realizara retiro justificado durante el primer semestre de 2018.

Como resultado de este proceso de selección se obtuvo información completa de 906 estudiantes del total de la población que fue de 1230 matriculados en el curso Matemática General.

\subsection{Técnicas de recolección}

Al inicio del primer semestre del año 2018 se le aplicó un cuestionario al estudiantado del curso Matemática General, en este se recolectó información sobre variables sociodemográficas y fue validado mediante una prueba piloto y un juicio de personas expertas en el tema. Las variables de tipo académico como la nota de admisión, la nota de colegio (en escalas de 0 a 100), la zona de procedencia y el estrato fueron proporcionadas por el Departamento de Registro de la UNA. Posteriormente, se unificaron los datos en una sola base que contenía la información de todos los participantes en el estudio.

\subsection{Procesamiento de análisis}

Primeramente, se realizó una descripción de las principales variables asociadas con el abandono escolar en el curso Matemática General. Seguidamente, con el fin de contrastar las hipótesis, se realizó una regresión logística múltiple, cuya expresión matemática para un modelo de $p$ variables independientes se expresa en la ecuación (1)

$$
P\left(Y=1 \mid X_{1}=x_{1}, \ldots, X_{p}=x_{p}\right)=\frac{e^{\beta_{0}+\beta_{1}+\cdots+\beta_{p}}}{1+e^{\beta_{0}+\beta_{1}+\cdots+\beta_{p}}}
$$

En los modelos de regresión logística es común calcular las razones de ventaja u odds ratio, que representa el cociente de la probabilidad de que un evento ocurra entre la probabilidad de que no ocurra, representado en la ecuación (2).

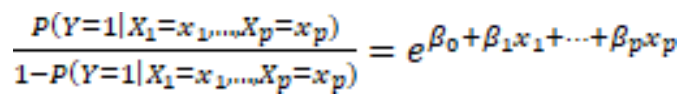

Para el caso en estudio, la variable dependiente $(Y)$ es la condición de abandono (1: SI; 0: NO) y las $p$ variables independientes están determinadas por la edad, el sexo de la persona estudiante, la tenencia de beca, la condición laboral, la carrera de ingreso, el 
estrato, la nota de colegio y la nota de admisión. Además, se utilizó el software estadístico R, versión 3.6.1, para los análisis estadísticos y el valor de 0,05 como nivel de significancia para la aceptación o rechazo de las hipótesis.

\section{Resultados}

Al realizar el análisis de las principales variables sociodemográficas, se logra determinar que la mayor cantidad de estudiantes que provienen del gran área metropolitana son mujeres, no poseen beca, son estudiantes a tiempo completo, provienen de colegios públicos, no tienen hijos y, en su mayoría, estudian carreras relacionadas con el área de las ciencias económicas (ver Tabla 1).

Tabla 1

UNA-Costa Rica. Distribución del estudiantado matriculado en el curso Matemática General según características sociodemográficas, I semestre 2018

\begin{tabular}{|c|c|c|c|}
\hline Variables & Categorización & $\begin{array}{l}\text { Número de } \\
\text { estudiantes }\end{array}$ & Porcentaje \\
\hline \multirow{2}{*}{ Sexo } & Hombre & 430 & 47,5 \\
\hline & Mujer & 476 & 52,5 \\
\hline \multirow{2}{*}{ Beca } & Sí & 344 & 38,0 \\
\hline & No & 562 & 62,0 \\
\hline \multirow{2}{*}{ Condición laboral } & Sí & 113 & 12,5 \\
\hline & No & 793 & 87,5 \\
\hline \multirow{2}{*}{ Zona } & GAM & 570 & 62,9 \\
\hline & No GAM & 336 & 37,1 \\
\hline \multirow{2}{*}{ Hijos o hijas } & Sí & 22 & 2,4 \\
\hline & No & 884 & 97,6 \\
\hline \multirow{3}{*}{ Estrato } & I & 221 & 24,4 \\
\hline & II & 553 & 61,0 \\
\hline & III & 132 & 14,6 \\
\hline \multirow{5}{*}{ Carrera } & Biología & 78 & 8,6 \\
\hline & Ciencias económicas & 516 & 57,0 \\
\hline & Educación & 68 & 7,5 \\
\hline & Geografía & 45 & 5,0 \\
\hline & Ingenierías & 199 & 22,0 \\
\hline
\end{tabular}

Fuente: elaboración propia 
De acuerdo con los datos de la Tabla 1, la mayor parte del estudiantado que matricula el curso de Matemática General cuenta con condiciones idóneas para tener un buen desempeño académico dado que no laboran, viven en la GAM y no poseen descendencia. Además, sus notas promedio en el examen de admisión y notas de colegio evidencian un rendimiento previo en la educación secundaria aceptable, según la nota mínima de calificación en el Ministerio de Educación Pública (MEP), y un buen rendimiento en la prueba de aptitud académica (ver Tabla 2).

En el modelo no se incluyó el número de hijos o hijas debido a que la mayoría del estudiantado no tenía progenie. Con respecto a la zona de procedencia, se consideró que la clasificación que realiza la UNA del estudiantado, en estratos, está muy relacionada con la zona de residencia, por lo que se decidió trabajar la variable estrato en vez de la zona de residencia debido a la importancia que representa a nivel institucional.

Tabla 2

UNA-Costa Rica. Estadísticas descriptivas de la edad y de las principales variables académicas del estudiantado matriculado en el curso Matemática General, I semestre 2018.

\section{Edad Nota de colegio Nota de admisión Número de veces que matriculó el curso*}

\begin{tabular}{|c|c|c|c|c|}
\hline Mínimo & 16 & 66,73 & 56,09 & 1 \\
\hline Máximo & 39 & 99,08 & 100 & 6 \\
\hline Mediana & 18 & 85,29 & 73,04 & 1 \\
\hline Moda & 18 & 80,83 & 75,35 & 1 \\
\hline Promedio & 18,91 & 85,11 & 73,55 & 1,385 \\
\hline $\begin{array}{l}\text { Desviación } \\
\text { estándar }\end{array}$ & 2,505 & 6,253 & 6,35 & 0,891 \\
\hline
\end{tabular}

*La cantidad de veces que la persona estudiante matriculó el curso antes del primer ciclo 2018. Fuente: elaboración propia.

La Tabla 3 muestra los coeficientes, errores estándar y valores $p$ para cada una de las variables independientes del modelo de regresión logística. Se considera que el modelo tiene un adecuado ajuste, pues tanto el test de Hosmer-Lemeshow $(p=0,227)$ como el test de razón de verosimilitud $(p<0,001)$ así lo sugieren. Además, se utilizó el factor de inflación de 
variancia en las variables explicativas, donde se obtienen valores entre 1,097 y 1,387, lo que sugiere que no hay problemas de multicolinealidad.

De esta manera, como se puede apreciar en la Tabla 3, las variables sexo, estrato, nota del colegio, beca, condición laboral y tipo de carrera resultaron significativas al 5\%, lo que indica la importancia de estas variables para explicar el abandono en el curso de Matemática General. Contrariamente la nota en el examen de admisión $(p=0,214)$ y la edad $(p=0,086)$ no resultan significativas (Tabla 3$)$.

Tabla 3

UNA-Costa Rica. Modelo de regresión logística para el abandono del estudiantado del curso Matemática General del I semestre 2018.

\begin{tabular}{|c|c|c|c|c|}
\hline Variables & Betas & Error estándar & Valor z & $\operatorname{Pr}(>|z|)$ \\
\hline (Intercepto) & 2,423 & 1,654 & 1,464 & 0,143 \\
\hline Sexo & 0,358 & 0,164 & 2,185 & $0,029^{\star}$ \\
\hline Estrato2 & 0,757 & 0,214 & 3,534 & $<0,001^{*}$ \\
\hline Estrato3 & 1,293 & 0,296 & 4,365 & $<0,001^{*}$ \\
\hline Nota de admisión & $-0,019$ & 0,015 & $-1,242$ & 0,214 \\
\hline Nota de colegio & $-0,055$ & 0,014 & $-3,783$ & $<0,001^{*}$ \\
\hline Ciencias Económicas² & 0,970 & 0,385 & 2,522 & $0,012^{\star}$ \\
\hline Geografía & 0,716 & 0,505 & 1,416 & 0,157 \\
\hline Educación & 0,628 & 0,536 & 1,17 & 0,242 \\
\hline Ingeniería & 1,312 & 0,396 & 3,308 & $0,001^{*}$ \\
\hline Beca & $-0,589$ & 0,1822 & $-3,231$ & $0,001^{*}$ \\
\hline Edad & 0,058 & 0,034 & 1,718 & 0,086 \\
\hline Condición laboral & 0,624 & 0,239 & 2,606 & $0,009^{*}$ \\
\hline
\end{tabular}

*coeficientes menores a 0,05

${ }^{1}$ Hombre fue la categoría de referencia.

²Biología fue la categoría de referencia para la variable carrera.

Fuente: elaboración propia.

Asimismo, la Figura 1 muestra los odds ratios o razones de ventaja para el modelo propuesto, en el que se puede determinar que la beca y la nota de colegio actúan como factores protectores, como originalmente se pensaba, lo que apoya las hipótesis de que las personas estudiantes becadas y con mayor puntaje en la nota del colegio tienen una menor posibilidad de abandono, comparado con las personas estudiantes no becadas y con menores puntajes en la nota de colegio. 
Figura 1

UNA-Costa Rica: Odds Ratio para el modelo de regresión logística de abandono en el curso Matemática General.

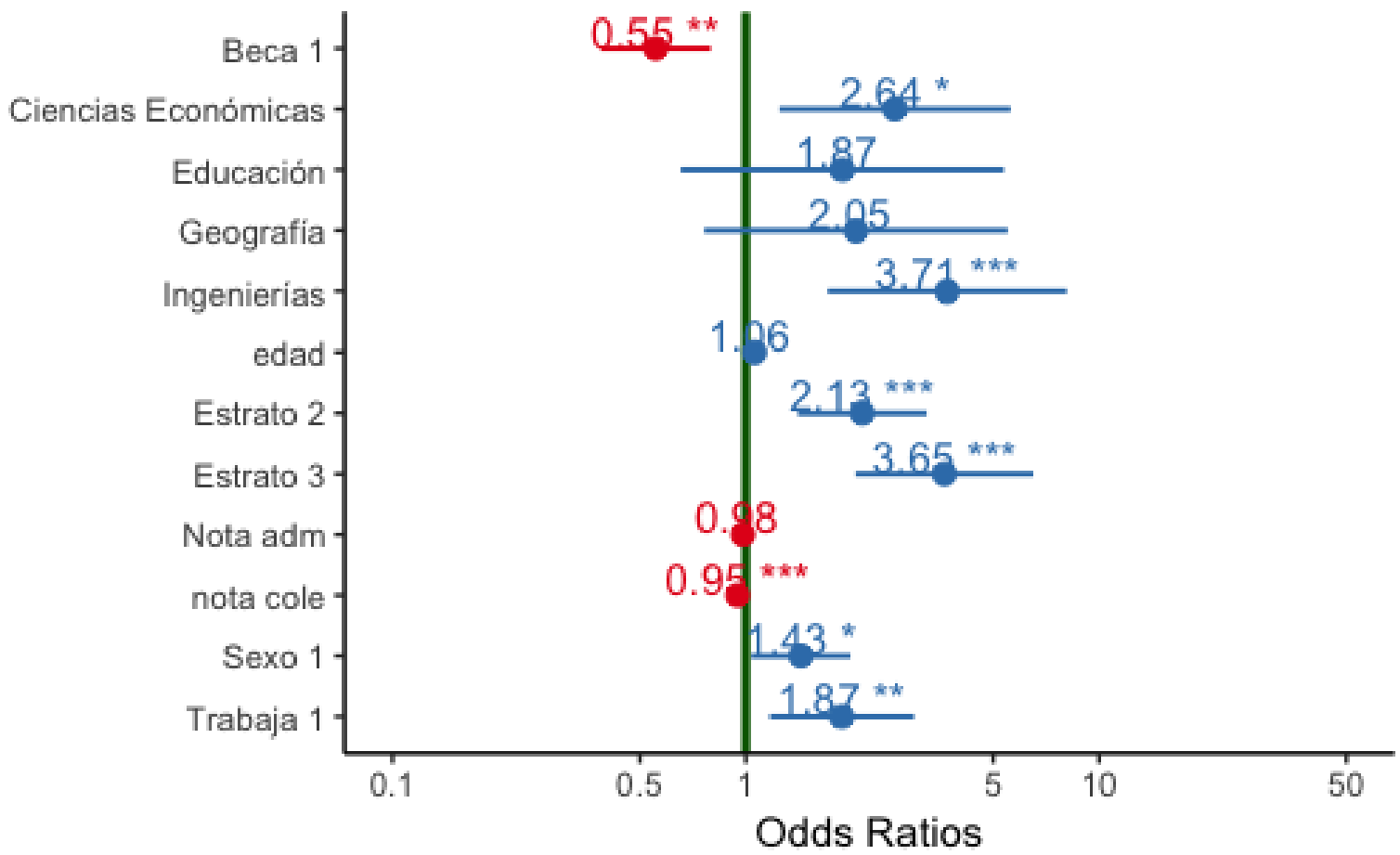

Nota: * coeficiente significativo al $0.001,{ }^{* *}$ coeficiente significativo al $0.01,{ }^{* * *}$ coeficiente significativo al 0.05

Fuente: elaboración propia.

En la Figura 1, los valores que están a la derecha de 1, en el eje $x$, representan aquellos factores que podrían ser considerados como de riesgo para el abandono estudiantil. Asimismo, los ubicados a la izquierda podrían ser clasificados como protectores. Aunque el objetivo del análisis es de tipo explicativo, el porcentaje de clasificación correcto del modelo es de 72,0\%; sin embargo, los coeficientes de determinación de Nargelkerke es de 0,182, McFadden 0,113 y CoxSnell 0,129 reflejan poco poder predictivo del modelo.

Por tanto, se procede a interpretar los coeficientes estadísticamente significativos, por ejemplo, si se mantienen las demás variables constantes, el riesgo de abandonar el curso de una persona estudiante que posee beca disminuye en un $45 \%$, comparado con una persona estudiante que no posee beca. Asimismo, por cada punto adicional en la calificación de la nota del colegio, el riesgo de abandono se reduce en un $5 \%$, lo que concuerda con los estudios previos realizados en la UNA (Rodríguez-Pineda y Zamora-Araya, 2014a; Rodríguez-Pineda y Zamora-Araya, 2014b; Rodríguez-Pineda, 2018). Por otro lado, el ser hombre y trabajar aumenta el riesgo de abandono en un $43 \%$ y $87 \%$, respectivamente, lo 
que coincide con resultados obtenidos en otras investigaciones sobre el abandono (Himmel, 2002; Rodríguez, Posada, Velásquez y Estrada, 2013; Santos, 2009; Vergara, Boj del Val, Barriga y Díaz, 2017).

En cuanto a la carrera, ser estudiante de ingeniería o ciencias económicas incrementa la posibilidad de abandonar el curso de Matemática General en 3,71 veces y 2,64 veces, respectivamente, con respecto a una persona estudiante del área de ciencias biológicas. Estos resultados son similares a los obtenidos en otros estudios donde el área de las ingenierías o carreras con alto contenido matemático presentan las mayores tasas de abandono (Campillo et al., 2018; Lázaro, Callejas, Griol y Durán, 2017; Rodríguez-Pineda y Zamora-Araya, 2014a).

No obstante, el resultado más revelador es el que comprueba la hipótesis de que el estrato III es el de mayor proporción de abandono, pues el riesgo se incrementa en 2,13 y 3,65 veces en estudiantes del estrato II y III, respectivamente, en comparación con estudiantes del estrato I. Esto muestra cómo, a pesar de los esfuerzos de la UNA por dar mayores posibilidades de acceso a la población con menores oportunidades educativas, en el curso de Matemática General se siguen evidenciando las diferencias basadas en la condición de los colegios de procedencia. Este resultado se une a otros estudios que evidencian que el tipo de colegio de procedencia y modalidad escolar recibida en la educación secundaria afectan la permanencia a nivel universitario (Gessaghi y Llinás, 2005; Rodríguez et al. 2013; Santos, 2009).

\section{Conclusiones}

Los resultados muestran que el sexo, el estrato, la nota de colegio, la beca, la carrera y la condición laboral pueden ser consideradas como variables relacionadas con el abandono en el curso de Matemática General. Lo anterior concuerda con otras investigaciones sobre el abandono realizadas tanto a nivel nacional como internacional (Gessaghi y Llinás, 2005; Himmel, 2002; Rodríguez-Pineda y Zamora-Araya, 2014a; Rodríguez-Pineda, 2018; Santos, 2009). Es así como se puede señalar que los hombres que cursan carreras del área de ingeniería, de estratos bajos y laboralmente activos son los más propensos a retirarse prematuramente de las aulas. Por su parte, las personas estudiantes becadas y con buenas notas de colegio tienen más posibilidades de concluir el curso.

No obstante, también hubo hipótesis relacionadas con la edad (a mayor edad mayor abandono) y con la nota del examen de admisión (a mayor nota menor abandono), cuyos 
coeficientes no resultaron estadísticamente significativos. Este resultado contrasta con los obtenidos por otras investigaciones, donde la edad y las notas obtenidas en las pruebas de admisión están negativa y positivamente correlacionadas con el abandono estudiantil respectivamente (Eckert y Suénaga, 2015; Rodríguez-Pineda y Zamora-Araya, 2014a; Rué, 2014, Torres, Rivera, Flores, Guadarrama y Dorantes, 2018, Zamora-Araya y VillalobosMadrigal, 2018b).

Esto podría deberse, en el caso de la primera variable, a que la muestra resultó ser muy homogénea en cuanto a edad se refiere, es decir, la mayor parte del estudiantado son jóvenes alrededor de 19 años (desviación estándar de 2,5) provenientes de la educación secundaria.

En cuanto a la nota del examen de admisión, aunque se esperaba que se asociara con el abandono, lo cierto es que no resultó de esta manera. Contrario a las notas del colegio, el examen de admisión representa una prueba de razonamiento, no de conocimientos, y el curso Matemática General está más enfocado a la adquisición de conocimientos que al razonamiento, lo que podría explicar el nivel de correlación de ambos constructos con la variable abandono.

Por otra parte, resulta de particular interés constatar cómo los estratos más vulnerables tienen mayor riesgo de abandono con respecto al estrato I, que representa al estudiantado con mejores oportunidades educativas previas. Esto muestra el peso que tienen las condiciones de educación previa en las personas estudiantes universitarias en cuanto a su deseo de persistir en cursos de nivel universitario en el área de matemática.

Los porcentajes de abandono para la muestra obtenida en el curso Matemática General en los estratos I, II y III son de 18,55\%, 30,56\% y 46,97\% respectivamente. Estas cifras muestran cómo conforme aumenta el número del estrato, aumenta la proporción de estudiantes que se retira de las aulas, lo que evidencia que las personas provenientes de los sectores con menores oportunidades educativas del país son los más afectados por el fenómeno del abandono escolar en el curso Matemática General de la UNA.

\section{Recomendaciones}

Los resultados obtenidos reflejan la necesidad de que la UNA tome acciones en beneficio de la permanencia del estudiantado de los estratos bajos en los cursos del área de matemática, en especial aquellos de los bloques iniciales, pues la mayor parte del abandono 
escolar tiene lugar en los primeros períodos lectivos (Canales y De los Ríos, 2018; Himmel, 2002; Rodríguez-Pineda y Zamora-Araya, 2014a; Santos, 2009).

En este particular, aunque la UNA ha hecho esfuerzos para que las personas estudiantes en situaciones de vulnerabilidad social ingresen a la institución (el sistema de admisión es un buen ejemplo de ello) se requiere de propuestas tendientes a disminuir el abandono en los cursos, sobre todo del área de matemática. Algunas propuestas que se pueden mencionar son:

a) Revisar el programa del curso de Matemática General, de forma tal que el curso brinde una adaptación al ambiente universitario teniendo en cuenta que muchos de los contenidos no se abordan en secundaria.

b) Fortalecer la figura del guía académico para dar un mejor acompañamiento a las personas estudiantes de primer ingreso a la Universidad.

c) Implementar un examen de ubicación, en cuanto a conocimientos matemáticos, que permita diagnosticar las debilidades y fortalezas con que ingresa el estudiantado.

d) Coordinar tutorías con el programa de Éxito Académico para dar prioridad en la matrícula al estudiantado de los estratos II y III considerando también las notas obtenidas en la prueba de ubicación.

e) Impulsar la implementación de investigaciones que realicen intervenciones de tipo metodológico y que permitan reducir la magnitud del fenómeno del abandono.

f) Valorar la modificación de la evaluación del curso, pues la nota se obtiene mediante el promedio de tres exámenes parciales igualmente ponderados.

A nivel universitario, se hace urgente tomar acciones dirigidas a disminuir el abandono en el curso de Matemática General. Se debe profundizar en las causas y hacer una revisión del programa del curso para atender las diferentes necesidades y condiciones del estudiantado, que incluirá aspectos como contenidos, recursos didácticos, técnicas y métodos de evaluación y estrategias pedagógicas, entre otros aspectos.

Paralelamente a esto, se debe trabajar en la puesta en marcha de estrategias para motivar al estudiantado, realizar un proceso de inducción a la vida universitaria de una forma integral y que incluya el uso y el manejo del tiempo, hábitos de estudio, tutorías, entre otros. Además, la ejecución de un proceso de nivelación orientado a solventar deficiencias que puedan tener en conocimientos previos, y que se consideran base para los cursos de matemática a nivel universitario. 
Lo cierto es que resulta indiscutible que se necesita revertir las cifras de abandono y, si bien no existe un camino evidente para hacerlo, la UNA en su afán de ofrecer oportunidades a los sectores más vulnerables, debe procurar encontrar la manera de cerrar las brechas sociales que se ven reflejadas en las aulas universitarias, sin perder con ello la calidad de la educación que brinda. Este, es sin duda alguna, el mayor reto que tienen las instituciones de educación superior en Costa Rica.

\section{Agradecimientos}

Se agradece al estudiantado y profesorado del curso Matemática General por su colaboración durante la recolección de información, así como a la Escuela de Matemática de la Universidad Nacional y a los proyectos Factores asociados a la deserción estudiantil en la carrera de Enseñanza de la Matemática de la UNA, código 0307-16, y La deserción y repitencia en el curso de Matemática General en la Universidad Nacional, código 0250-15.

\section{Referencias}

Avdic, Daniel y Gartell, Marie. (2015). Working while studying? Student aid design and socioeconomic achievement disparities in higher education. Labour Economics, 33, 2640. doi: 10.1016/j.labeco.2015.01.002

Braxton, John; Milem, Jeffrey y Sullivan, Anna. (2000). The influence of active learning on the college student departure process: Toward a revision of Tinto's theory. The Journal of Higher Education, 71(5), 569-590. doi: 10.2307/2649260

Burbano García, Claudia; Lozano Ramos, Carolina; Medina, Luisa y Guerrero, Yonni. (2015). Análisis de Factores Psicológicos, Hábitos y Técnicas de estudio asociados con el bajo rendimiento académico en un grupo de estudiantes de la Fundación Universitaria María Cano. Congresos CLABES. Recuperado de http://revistas.utp.ac.pa/index.php/clabes/article/view/1629

Cabrera, Alberto; Nora, Amaury y Castaneda, María. (1993). College Persistence: Structural Equations Modeling Test of an Integrated Model of Student Retention. Journal of Higher Education, 64(2), 123-139. Recuperado de https://doi.org/10.1080/00221546.1993.11778419

Campillo, Magda; García, Manuel; Hernández, Flora; Martínez, Adrián y Sánchez, Melchor. (2018). Factores Asociados a la no conclusión de la Licenciatura: la carrera de elección, un panorama histórico de 22 Años en la Universidad Nacional Autónoma de México. Congresos CLABES. Recuperado de http://revistas.utp.ac.pa/index.php/clabes/article/view/1902 
Canales, Andrea y De los Ríos, Danae. (2007). Factores explicativos de la deserción universitaria. Calidad en la Educación, (26). doi: http://dx.doi.org/10.31619/cale du.n26.239

Castaño, Elkin; Gallón, Santiago; Gómez, Karoll y Vásquez, Johanna. (2006). Análisis de los factores asociados a la deserción y graduación estudiantil universitaria. Lecturas de Economía (65), 9-35. Recuperado de https://www.redalyc.org/articulo.oa?id=155213357001

Crawford, Claire. (2014). Socio-economic differences in university outcomes in the UK: dropout, degree completion and degree class. Institute for Fiscal Studies. doi 10.1920/wp.ifs.2014.1431

Díaz-Mujica, Alejandro; García, Diego; López, Yaranay; Maluenda, Jorge; Hernández, Hebe y Pérez-Villalobos, María Victoria. (2018). Mediación del ajuste académico entre variables cognitivo-motivacionales y la intención de abandono en primer año de universidad. Congresos CLABES. Recuperado de http://revistas.utp.ac.pa/index.php/clabes/article/view/1934

Eckert, Karina y Suénaga, Roberto. (2015). Análisis de deserción-permanencia de estudiantes universitarios utilizando técnica de clasificación en minería de datos. Formación universitaria, 8(5), 03-12. Recuperado de http://dx.doi.org/10.4067/S0718$\underline{50062015000500002}$

Esteban, María; Bernardo, Ana y Rodríguez-Muñiz, Luis. (2016). Permanencia en la universidad: la importancia de un buen comienzo. Aula abierta, 44(1), 1-6. Recuperado de http://dx.doi.org/10.1016/i.aula.2015.04.001

Gamboa, Ronny; Castillo, Mario e Hidalgo, Randall. (2019). Errores matemáticos de estudiantes que ingresan a la universidad. Actualidades Investigativas en Educación, 19(1), 31-31. doi 10.15517/aie.v19i1.35278

Gessaghi, Victoria y Llinás, Paola. (2005). Democratizar el acceso a la educación superior [pdf]. Centro de Implementación de Políticas Públicas para la Equidad y el Crecimiento. Recuperado de https://www.cippec.org/publicacion/democratizar-el-acceso-a-laeducacion-superior/

González, Luis; Uribe, Daniel y González, Soledad. (2005). Estudio sobre la repitencia y deserción en la educación superior chilena. [pdf]. Estudio IESALC, Chile. Recuperado de

https://www.inacap.cl/tportal/portales/tp4964b0e1bk102/uploadlmg/File/REPITENCIA DESERCION L E Gonzalez 2005.pdf

Henríquez, Natalia y Escobar, Diego. (2016). Construcción de un modelo de alerta temprana para la detección de estudiantes en riesgo de deserción de la Universidad Metropolitana de Ciencias de la Educación. Revista mexicana de investigación educativa, 21(71), 1221-1248. Recuperado de http://www.scielo.org.mx/scielo.php?script=sci arttext\&pid=S1405$66662016000401221 \& \operatorname{lng}=\mathrm{es} \& \ln \mathrm{n}=\mathrm{es}$ 
Himmel, Erika. (2002). Modelos de análisis de la deserción estudiantil en la educación superior. Revista Calidad de Educación, (17). doi: https://doi.org/10.31619/caledu.n17.409

International Institute for Higher Education in Latin America (IESALC). (2006). Informe sobre la educación superior en América Latina y el Caribe, 2000-2005: la metamorfosis de la educación superior. $\quad$ [pdf]. Recuperado de https://www.mineducacion.gov.co/cvn/1665/articles-100800 archivo pdf.pdf

Lázaro, Niursys; Callejas, Zoraida; Griol, David y Durán, Mayra. (2017). La deserción estudiantil en educación superior: SOS en carreras de ingeniería informática. Congresos CLABES. Recuperado de http://revistas.utp.ac.pa/index.php/clabes/article/view/1674

Páramo, Gabriel y Correa, Carlos. (1999). Deserción estudiantil universitaria. Conceptualización. Revista Universidad EAFIT, 114, 65-78. [pdf]. Recuperado de http://publicaciones.eafit.edu.co/index.php/revista-universidadeafit/article/view/1075/967

Pascua-Cantarero, Paola. (2016). Factores relacionados con la deserción en el primer y segundo año de estudio en la carrera de Enseñanza de la Matemática de la Universidad Nacional de Costa Rica. Revista Electrónica Educare, 20(1), 1-23. http://dx.doi.org/10.15359/ree.20-1.5

Ramírez, Carolina. (2013). Deserción del estudiantado de la carrera de Psicología de la Universidad Nacional, durante el período 2007 al 2011 y análisis de factores que inciden en esa deserción, como sustento para el diseño de una propuesta de mejoramiento (Proyecto de Graduación para optar el grado de Maestría en Educación). Universidad de Costa Rica, San José, Costa Rica.

Rodríguez-Pineda, Magaly. (2018). De la reproducción social en el acceso y la permanencia universitaria. Caso de la Universidad Nacional de Costa Rica. Período 2009-2016 (Tesis de maestría). Universidad de Costa Rica, San José, Costa Rica.

Rodríguez-Pineda, Magaly y Zamora-Araya, José. (2014a). Análisis de la deserción en la Universidad Nacional desde una perspectiva longitudinal. Ponencia preparada para el Quinto Informe Estado de la Educación. San José: Programa Estado de la Nación. Recuperado https://estadonacion.or.cr/files/biblioteca virtual/educacion/005/Magaly Rodriguez Ana lisis de la desercion.pdf

Rodríguez-Pineda, Magaly y Zamora-Araya, José. (2014b). Operacionalización de la permanencia estudiantil en las carreras de pregrado y grado de la Universidad Nacional. Ponencia preparada para el Quinto Informe Estado de la Educación. San José: Programa Estado de la Nación. Recuperado de https://estadonacion.or.cr/files/biblioteca virtual/educacion/005/Magaly Rodriguez Ope racionalizacion de la permanencia.pdf 
Rodríguez, Marco; Posada, Margarita; Velásquez, Melbin y Estrada, Patricia. (2013). Población con riesgo de abandono universitario. Una aproximación desde la prevención. Congresos CLABES. Recuperado de http://revistas.utp.ac.pa/index.php/clabes/article/view/873

Rué, Joan. (2014). El abandono universitario: variables, marcos de referencia y políticas de calidad. REDU. Revista de docencia universitaria, 12(2), 281-306. Recuperado de https://doi.org/10.4995/redu.2014.5649

Sandoval, Iván; Sánchez, Tarquino; Velasteguí, Viviana y Naranjo, David. (2018). Factores asociados al Abandono en estudiantes de grupos vulnerables. Caso Escuela Politécnica Nacional. Congresos CLABES. Recuperado de http://revistas.utp.ac.pa/index.php/clabes/article/view/1907/2850

Santos, Humberto. (2009). Dinámica de la deserción escolar en Chile. Santiago: Centro de Políticas Comparadas de Educación (CPCE). Recuperado de http://www.ministeriodesarrollosocial.gob.cl/btca/txtcompleto/mideplan/ser.estsocdinamdeserc.escolar.pdf

Tinto, Vincent. (1982). Defining dropout: A matter of perspective. New Directions for Institutional Research, (36), https://doi.org/10.1002/ir.37019823603 
Zamora-Araya, José y Villalobos-Madrigal, Francisco. (2018a). El Enfoque Por Competencias en la Carrera de Enseñanza de la Matemática de la Universidad Nacional y su Relación con el abandono escolar. Congresos CLABES. Recuperado de http://revistas.utp.ac.pa/index.php/clabes/article/view/1897

Zamora-Araya, José y Villalobos-Madrigal, Francisco. (2018b). Factors associated with dropping out of the program for Bachelor's and Licentiate's Degrees in Mathematics Teaching at the Universidad Nacional de Costa Rica (UNA): Evidence from the 2016 Student Cohort. Uniciencia, 32(2), 111-126. Recuperado de http://dx.doi.org/10.15359/ru.32-2.8 
Revista indizada en

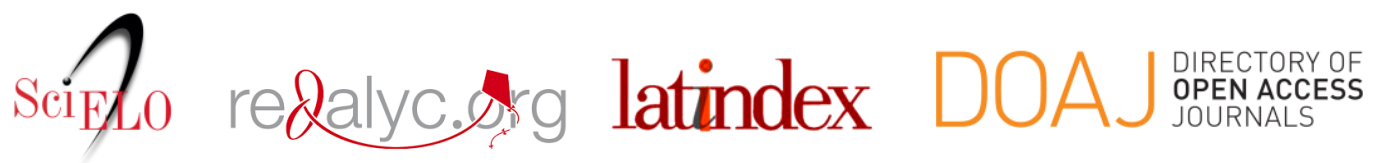

Distribuida en las bases de datos:

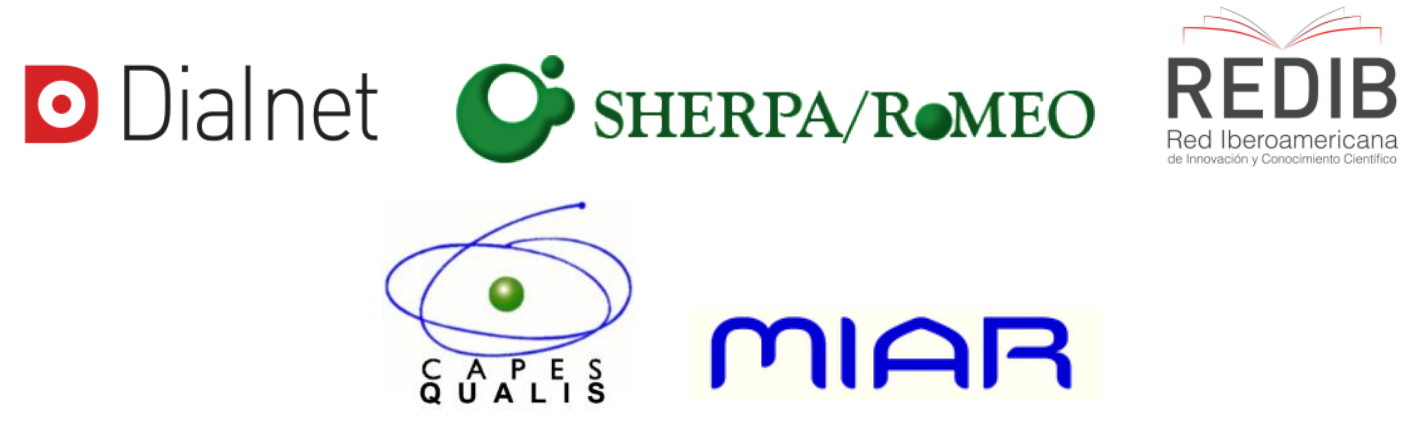

